\title{
DERIVATIONS IN LUKASIEWICZ SEMIRINGS
}

\author{
IVAN CHAJDA AND HELMUT LÄNGER
}

Received 29 May, 2018

\begin{abstract}
An axiomatization of classical propositional logic is provided by means of Boolean algebras which are term equivalent to Boolean rings. This is important because rings form a classical part of algebra whose tools can be used for the investigations. The Łukasiewicz manyvalued logic was axiomatized via so-called MV-algebras by C. C. Chang in 1950's. MV-algebras are successfully applied in the logic of quantum mechanics and hence they are considered as quantum structures. It is a natural question if also MV-algebras have their alter ego among classical structures. For this reason the so-called Łukasiewicz semirings were introduced by the first author and his collaborators in [3] - [4]. As shown, Łukasiewicz semirings are term equivalent to MV-algebras and we can use with advantage several developed tools for their study. In particular, we investigate derivations in semirings which were introduced formerly but here these semirings are enriched by an involution.
\end{abstract}

2010 Mathematics Subject Classification: 13N15; 16Y60; 06D35

Keywords: Łukasiewicz semiring, MV-algebra, derivation

\section{INTRODUCTION}

The concept of derivation in rings was introduced already in the 1960's. For semirings, it was defined in J. S. Golan's book ([9]). However, derivations were studied also in lattices (see e.g. [7] and [11]). Let us note that bounded distributive lattices are special semirings. Starting with 2010, several authors extended the study of derivations to certain algebras forming an algebraic semantic for non-classical logics including the logic of quantum mechanics. In this context let us mention the papers [1] and [10]. It was shown in [5] that every MV-algebra can be converted into a socalled Łukasiewicz semiring. The advantage of this approach is that we can apply also methods from the theory of semirings. This was done by the authors in [6].

The main difference between our approach to derivations in Łukasiewicz semirings and the approach by J. S. Golan in [9] is that in Łukasiewicz semirings we have a unary operation such that addition can be expressed by both multiplication and this

Support of the research of both authors by ÖAD, project CZ 04/2017, of the first author by IGA, project PřF 2018 012, and of the second author by the Austrian Science Fund (FWF), project I 1923$\mathrm{N} 25$, is gratefully acknowledged. 
unary operation. Moreover, Łukasiewicz semirings are additively idempotent and ordered by the induced semilattice ordering, see [1] - [4]. On the other hand, these semirings are not lattices and hence we cannot use results and methods from [7] or [11].

\section{2. ŁUKASIEWICZ SEMIRINGS}

We start with the following definition:

Definition 1. A Eukasiewicz semiring is an algebra $\mathbf{R}=\left(R,+, \cdot,{ }^{\prime}, 0,1\right)$ of type $(2,2,1,0,0)$ such that

- $(R,+, 0)$ is a commutative monoid,

- $(R, \cdot, 1)$ is a commutative monoid,

- $(x+y) \cdot z \approx x \cdot z+y \cdot z$

- $x \cdot 0 \approx 0$,

- $x+x \approx x$ and $x+1 \approx 1$,

- $\left(x^{\prime} \cdot y\right)^{\prime} \cdot y \approx\left(y^{\prime} \cdot x\right)^{\prime} \cdot x$,

- $x \leq y$ implies $y^{\prime} \leq x^{\prime}$,

- $\left(x^{\prime}\right)^{\prime} \approx x$.

An algebra $(R,+, \cdot, 0,1)$ of type $(2,2,0,0)$ satisfying the first four conditions is called a commutative semiring. So $(R,+)$ can be considered a join-semilattice and we denote by $\leq$ its corresponding partial order relation. We call it the induced order of R. Put

$$
x \wedge y:=\left(x^{\prime}+y^{\prime}\right)^{\prime}
$$

for all $x, y \in R$. Since $(R,+, 0,1)$ is a bounded join-semilattice and ' is an antitone involution on $(R, \leq),(R,+, \wedge, 0,1)$ is a bounded lattice.

Example 1. The algebra $\mathbf{R}=\left([0,1], \vee, \odot,^{\prime}, 0,1\right)$ with

$$
\begin{aligned}
x \vee y & :=\max (x, y), \\
x \odot y & :=(x+y-1) \vee 0, \\
x^{\prime} & :=1-x
\end{aligned}
$$

for all $x, y \in[0,1]$ is a Łukasiewicz semiring. Here $[0,1]$ denotes the unit interval of the real numbers.

Example 2. For every positive integer $n$ the algebra $\mathbf{R}_{n}=(\{0,1,2, \ldots, n\}, \vee, \odot$, $', 0, n)$ with

$$
\begin{aligned}
x \vee y & :=\max (x, y), \\
x \odot y & :=(x+y-n) \vee 0, \\
x^{\prime} & :=n-x
\end{aligned}
$$

for all $x, y=0, \ldots, n$ is a Łukasiewicz semiring. Note that in this case the greatest element is $n$. 
Example 3. Every Boolean algebra $\mathbf{B}=\left(B, \vee, \wedge,{ }^{\prime}, 0,1\right)$ is a Łukasiewicz semiring.

Lemma 1. Let $\mathbf{R}=\left(R,+, \cdot{ }^{\prime}, 0,1\right)$ be a tukasiewicz semiring and $a, b, c \in R$. Then

(i) $0^{\prime}=1$ and $1^{\prime}=0$,

(ii) $a \cdot a^{\prime}=0$,

(iii) $a \leq b$ implies $a \cdot c \leq b \cdot c$, and hence $a \cdot b \leq a \wedge b$, especially, $a \cdot a \leq a$,

(iv) $a \cdot(a+b)^{\prime}=0$,

(v) $a+b=\left(\left(a \cdot b^{\prime}\right)^{\prime} \cdot b^{\prime}\right)^{\prime}$,

(vi) $a \leq b$ if and only if $a \cdot b^{\prime}=0$.

Proof.

(i) This follows immediately from the fact that 0 is the smallest and 1 the greatest element of $(R, \leq)$ and that ' is an antitone involution of $(R, \leq)$.

(ii) According to (i) we have $a \cdot a^{\prime}=\left(1 \cdot a^{\prime}\right)^{\prime} \cdot a^{\prime}=(a \cdot 0)^{\prime} \cdot 0=0$.

(iii) $a \leq b$ implies $a+b=b$ whence $a \cdot c+b \cdot c=(a+b) \cdot c=b \cdot c$, i.e. $a \cdot c \leq b \cdot c$. Since $a \cdot b \leq a \cdot 1=a$ and $a \cdot b \leq 1 \cdot b=b$, we have $a \cdot b \leq a \wedge b$.

(iv) According to (iii) and (ii) we have $a \cdot(a+b)^{\prime} \leq(a+b) \cdot(a+b)^{\prime}=0$ and hence $a \cdot(a+b)^{\prime}=0$.

(v) According to (iv), (i) and (ii) we have

$a+b=\left(\left(a \cdot(a+b)^{\prime}\right)^{\prime} \cdot(a+b)^{\prime}\right)^{\prime}=\left(\left((a+b) \cdot a^{\prime}\right)^{\prime} \cdot a^{\prime}\right)^{\prime}=\left(\left(b \cdot a^{\prime}\right)^{\prime} \cdot a^{\prime}\right)^{\prime}=\left(\left(a \cdot b^{\prime}\right)^{\prime} \cdot b^{\prime}\right)^{\prime}$.

(vi) If $a \leq b$ then according to (iii) and (ii) we have $a \cdot b^{\prime} \leq b \cdot b^{\prime}=0$ showing $a \cdot b^{\prime}=0$. If, conversely $a \cdot b^{\prime}=0$ then according to (v) and (i) we have $a \leq a+b=\left(\left(a \cdot b^{\prime}\right)^{\prime} \cdot b^{\prime}\right)^{\prime}=b$.

An element $a$ of $R$ is called Boolean if $a \cdot a=a$. This is equivalent to $a+a^{\prime}=1$ and to $a \wedge a^{\prime}=0$. Hence, $a$ is Boolean if and only if $a^{\prime}$ has this property. The mentioned equivalence can be seen as follows: If $a \cdot a=a$ then $a+a^{\prime}=\left(\left(a \cdot\left(a^{\prime}\right)^{\prime}\right)^{\prime}\right.$. $\left.\left(a^{\prime}\right)^{\prime}\right)^{\prime}=\left((a \cdot a)^{\prime} \cdot a\right)^{\prime}=\left(a^{\prime} \cdot a\right)^{\prime}=0^{\prime}=1$. If, conversely, $a+a^{\prime}=1$ then $a \cdot a=$ $a \cdot a+0=a \cdot a+a \cdot a^{\prime}=a \cdot\left(a+a^{\prime}\right)=a \cdot 1=a$. Let Bool $\mathbf{R}$ denote the set of all Boolean elements of $R$. It is easy to see that Bool $\mathbf{R}$ is a subuniverse of $\left(R, \cdot{ }^{\prime}, 0,1\right)$.

An $M V$-algebra is an algebra $(A, \oplus, \neg, 0)$ of type $(2,1,0)$ satisfying the following identities:

$$
\begin{aligned}
(x \oplus y) \oplus z & \approx x \oplus(y \oplus z), \\
x \oplus y & \approx y \oplus x, \\
x \oplus 0 & \approx x, \\
x \oplus 1 & \approx 1, \\
\neg(\neg x) & \approx x,
\end{aligned}
$$


Here and in the following $1:=\neg 0$. We define

$$
\begin{aligned}
x \otimes y & :=\neg(\neg x \oplus \neg y), \\
x \vee y & :=\neg(\neg x \oplus y) \oplus y, \\
x \wedge y & :=\neg(\neg x \vee \neg y)
\end{aligned}
$$

for all $x, y \in A$. Then $\otimes$ is distributive with respect to $\vee$. Moreover, for $x, y \in A$ we define $x \leq y$ if $\neg x \oplus y=1$. Then $(A, \vee, \wedge, 0,1)$ is a bounded distributive lattice whose induced order coincides with $\leq$. Moreover $x \leq y$ implies $\neg y \leq \neg x(x, y \in A)$.

It is known that Łukasiewicz semirings are in a natural one-to-one correspondence with MV-algebras (cf. e.g. [2], [3] and [8]). For the convenience of the reader we provide a short direct proof.

Theorem 1. If $\mathbf{R}=\left(R,+, \cdot,{ }^{\prime}, 0,1\right)$ is a Eukasiewicz semiring and

$$
x \oplus y:=\left(x^{\prime} \cdot y^{\prime}\right)^{\prime}
$$

for all $x, y \in R$ then $\mathrm{M}(\mathbf{R}):=\left(R, \oplus,{ }^{\prime}, 0\right)$ is an MV-algebra. If, conversely, $\mathbf{A}=$ $(A, \oplus, \neg, 0)$ is an MV-algebra then $\mathbb{R}(\mathbf{A}):=(A, \vee, \otimes, \neg, 0,1)$ is a Eukasiewicz semiring. This correspondence is one-to-one.

Proof. First assume $\mathbf{R}=\left(R,+, \cdot{ }^{\prime}, 0,1\right)$ to be a Łukasiewicz semiring and define $x \oplus y:=\left(x^{\prime} \cdot y^{\prime}\right)^{\prime}$ for all $x, y \in R$. Then we have

$$
\begin{aligned}
(x \oplus y) \oplus z & \approx\left(\left(\left(x^{\prime} \cdot y^{\prime}\right)^{\prime}\right)^{\prime} \cdot z^{\prime}\right)^{\prime} \approx\left(\left(x^{\prime} \cdot y^{\prime}\right) \cdot z^{\prime}\right)^{\prime} \approx\left(x^{\prime} \cdot\left(y^{\prime} \cdot z^{\prime}\right)\right)^{\prime} \approx \\
& \approx\left(x^{\prime} \cdot\left(\left(y^{\prime} \cdot z^{\prime}\right)^{\prime}\right)^{\prime}\right)^{\prime} \approx x \oplus(y \oplus z), \\
x \oplus y & \approx\left(x^{\prime} \cdot y^{\prime}\right)^{\prime} \approx\left(y^{\prime} \cdot x^{\prime}\right)^{\prime} \approx y \oplus x, \\
x \oplus 0 & \approx\left(x^{\prime} \cdot 0^{\prime}\right)^{\prime} \approx\left(x^{\prime} \cdot 1\right)^{\prime} \approx\left(x^{\prime}\right)^{\prime} \approx x, \\
x \oplus 1 & \approx\left(x^{\prime} \cdot 1^{\prime}\right)^{\prime} \approx\left(x^{\prime} \cdot 0\right)^{\prime} \approx 0^{\prime} \approx 1, \\
\left(x^{\prime}\right)^{\prime} & \approx x, \\
\left(x^{\prime} \oplus y\right)^{\prime} \oplus y & \approx\left(\left(\left(\left(\left(x^{\prime}\right)^{\prime} \cdot y^{\prime}\right)^{\prime}\right)^{\prime}\right)^{\prime} \cdot y^{\prime}\right)^{\prime} \approx\left(\left(\left(x^{\prime}\right)^{\prime} \cdot y^{\prime}\right)^{\prime} \cdot y^{\prime}\right)^{\prime} \approx\left(\left(\left(y^{\prime}\right)^{\prime} \cdot x^{\prime}\right)^{\prime} \cdot x^{\prime}\right)^{\prime} \approx \\
& \approx\left(\left(\left(\left(\left(y^{\prime}\right)^{\prime} \cdot x^{\prime}\right)^{\prime}\right)^{\prime}\right)^{\prime} \cdot x^{\prime}\right)^{\prime} \approx\left(y^{\prime} \oplus x\right)^{\prime} \oplus x .
\end{aligned}
$$

Hence $\mathbf{M}(\mathbf{R})=\left(R, \oplus,{ }^{\prime}, 0\right)$ is an $\mathrm{MV}$-algebra. Conversely, let $\mathbf{A}=(A, \oplus, \neg, 0)$ be an $\mathrm{MV}$-algebra. Then $(A, \vee, 0)$ is a commutative monoid. Moreover,

$$
\begin{aligned}
(x \otimes y) \otimes z & \approx \neg(\neg(\neg(\neg x \oplus \neg y)) \oplus \neg z) \approx \neg((\neg x \oplus \neg y) \oplus \neg z) \approx \\
& \approx \neg(\neg x \oplus(\neg y \oplus \neg z)) \approx \neg(\neg x \oplus \neg(\neg(\neg y \oplus \neg z))) \approx x \otimes(y \otimes z), \\
x \otimes y & \approx \neg(\neg x \oplus \neg y) \approx \neg(\neg y \oplus \neg x) \approx y \otimes x, \\
x \otimes 1 & \approx \neg(\neg x \oplus \neg 1) \approx \neg(\neg x \oplus 0) \approx \neg(\neg x) \approx x .
\end{aligned}
$$

Hence, $(A, \otimes, 1)$ is a commutative monoid, too. It is clear that the partial order relations in $\mathbf{A}$ and $\mathbb{R}(\mathbf{A})$ coincide. Now for $a, b \in A, a \leq b$ implies $\neg b \leq \neg a$. Moreover, 
we have

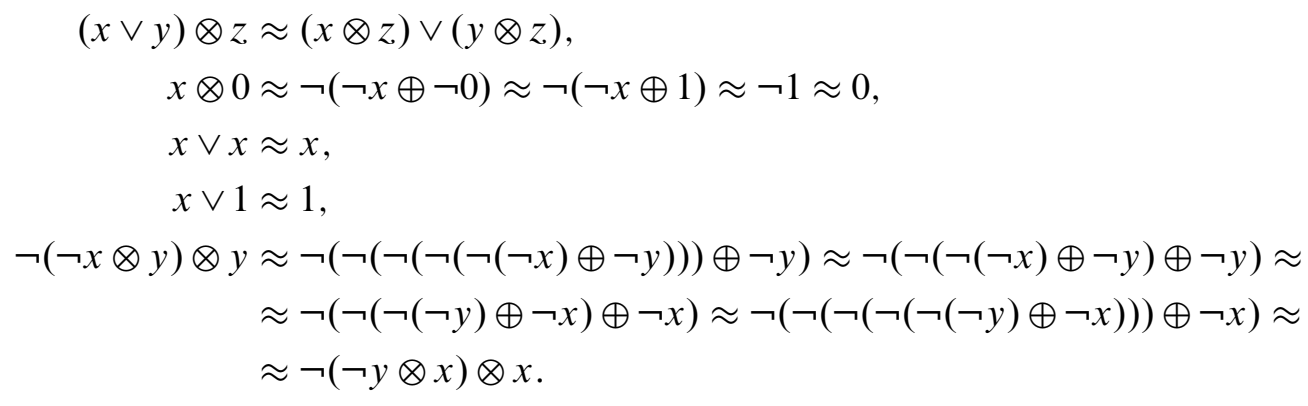

This shows that $\mathbb{R}(\mathbf{A})=(A, \vee, \otimes, \neg, 0,1)$ is a Łukasiewicz semiring. If $\mathbf{R}=\left(R,+, \cdot{ }^{\prime}, 0,1\right)$ is a Łukasiewicz semiring, $\mathbb{M}(\mathbf{R}):=\left(R, \oplus,{ }^{\prime}, 0\right)$ and $\mathbb{R}(\mathbf{M}(\mathbf{R}))=$ $\left(R, \vee, \otimes,^{\prime}, 0,0^{\prime}\right)$ then

$$
\begin{aligned}
x \vee y & \left.\approx\left(x^{\prime} \oplus y\right)^{\prime} \oplus y \approx\left(\left(\left(\left(x^{\prime}\right)^{\prime} \cdot y^{\prime}\right)^{\prime}\right)^{\prime}\right)^{\prime} \cdot y^{\prime}\right)^{\prime} \approx\left(\left(x \cdot y^{\prime}\right)^{\prime} \cdot y^{\prime}\right)^{\prime} \approx x+y, \\
x \otimes y & \approx\left(x^{\prime} \oplus y^{\prime}\right)^{\prime} \approx\left(\left(\left(x^{\prime}\right)^{\prime} \cdot\left(y^{\prime}\right)^{\prime}\right)^{\prime}\right)^{\prime} \approx x \cdot y, \\
0^{\prime} & \approx 1,
\end{aligned}
$$

i.e. $\quad \mathbb{R}(\mathbb{M}(\mathbf{R}))=\mathbf{R}$. If, finally, $\mathbf{A}=(A, \oplus, \neg, 0)$ is an MV-algebra, $\mathbb{R}(\mathbf{A}):=(A, \vee, \otimes, \neg, 0,1)$ and $\mathbb{M}(\mathbb{R}(\mathbf{A}))=\left(\left(A, \oplus_{1}, \neg, 0\right)\right.$ then

$$
x \oplus_{1} y \approx \neg(\neg x \otimes \neg y) \approx \neg(\neg(\neg(\neg x) \oplus \neg(\neg y))) \approx x \oplus y
$$

and hence $\mathbf{M}(\mathbb{R}(\mathbf{A}))=\mathbf{A}$.

Due to this correspondence, for every Łukasiewicz semiring $\mathbf{R}=\left(R,+, \cdot{ }^{\prime}, 0,1\right)$, $(R,+, \wedge, 0,1)$ is a bounded distributive lattice.

We are going to show when an interval in a Łukasiewicz semiring can be converted into a bounded lattice with an antitone involution.

Lemma 2. Let $\mathbf{R}=\left(R,+, \cdot{ }^{\prime}, 0,1\right)$ be a Eukasiewicz semiring and $b \in \mathrm{Bool} \mathbf{R}$ and put

$$
\begin{aligned}
x^{*} & :=x^{\prime} \wedge b, \\
x \cap y & :=\left(x^{*}+y^{*}\right)^{*}
\end{aligned}
$$

for all $x, y \in[0, b]$. Then $\left([0, b],+, \cap,{ }^{*}, 0, b\right)$ is a bounded lattice with an antitone involution.

Proof. Since $b \in$ Bool R, i.e. $b \wedge b^{\prime}=0$, we have

$$
\left(x^{*}\right)^{*}=\left(x^{\prime} \wedge b\right)^{\prime} \wedge b=\left(x+b^{\prime}\right) \wedge b=(x \wedge b)+\left(b^{\prime} \wedge b\right)=x+0=x
$$

for all $x \in[0, b]$. Obviously, ${ }^{*}$ is antitone. The rest of the proof is clear. 
Lemma 3. Let $\mathbf{R}=\left(R,+, \cdot{ }^{\prime}, 0,1\right)$ be a Eukasiewicz semiring and $a \in \mathrm{Bool} \mathbf{R}$ and put

$$
\begin{aligned}
x^{*} & :=x^{\prime}+a, \\
x \cap y & :=\left(x^{*}+y^{*}\right)^{*}
\end{aligned}
$$

for all $x, y \in[a, 1]$. Then $\left([a, 1],+, \cap,{ }^{*}, a, 1\right)$ is a bounded lattice with an antitone involution.

Proof. Since $a \in$ Bool R, i.e. $a+a^{\prime}=1$, we have

$$
\left(x^{*}\right)^{*}=\left(x^{\prime}+a\right)^{\prime}+a=\left(x \wedge a^{\prime}\right)+a=(x+a) \wedge\left(a^{\prime}+a\right)=x \wedge 1=x
$$

for all $x \in[a, 1]$. Obviously, ${ }^{*}$ is antitone. The rest of the proof is clear.

Theorem 2. Let $\mathbf{R}=\left(R,+, \cdot{ }^{\prime}, 0,1\right)$ be a Eukasiewicz semiring and $a, b \in \mathrm{Bool} \mathbf{R}$, assume $a \leq b$ and put

$$
\begin{aligned}
x^{*} & :=\left(x^{\prime}+a\right) \wedge b, \\
x \cap y & :=\left(x^{*}+y^{*}\right)^{*}
\end{aligned}
$$

for all $x, y \in[a, b]$. Then $\left([a, b],+, \cap,{ }^{*}, a, b\right)$ is a bounded lattice with an antitone involution.

Proof. This follows from the fact that $(R,+, \wedge)$ is a distributive lattice.

Remark 1. Theorem 2 remains valid if one defines $x^{*}:=\left(x^{\prime} \wedge b\right)+a$ for all $x \in$ $[a, b]$. Note that $\left(x^{\prime} \wedge b\right)+a \leq\left(x^{\prime}+a\right) \wedge b$ for all $x \in R$.

\section{DERIVATIONS}

Although Łukasiewicz semirings are in a one-to-one correspondence with MValgebras, we do not define derivations like in [1] or [10] since there the MV-operation $\oplus$ is used instead of + . Hence also our results differ essentially from those obtained in the mentioned papers.

Now we can adopt the definition of a derivation from [9].

Definition 2. Let $\mathbf{R}=\left(R,+, \cdot,{ }^{\prime}, 0,1\right)$ be a Łukasiewicz semiring. A derivation on $\mathbf{R}$ is a mapping $d$ from $R$ to $R$ satisfying the following identities:

$$
\begin{aligned}
d(x+y) & \approx d x+d y, \\
d(x \cdot y) & \approx(d x) \cdot y+x \cdot(d y) .
\end{aligned}
$$

Let Der $\mathbf{R}$ denote the set of all derivations on $\mathbf{R}$, for every $a \in R$ let $\bar{d}_{a}$ denote the mapping from $R$ to $R$ defined by $\bar{d}_{a}(x):=a \cdot x$ for all $x \in R$ and let id denote the identical mapping from $R$ to $R$. We call a derivation $d$ on $\mathbf{R}$ principal if it is of the form $\bar{d}_{a}$ for some $a \in R$. Hence $0=\bar{d}_{0}$ and id $=\bar{d}_{1}$ are principal derivations. For any mapping $d$ from $R$ to $R$ define Fix $d:=\{x \in R \mid d x=x\}$. 
Here and in the following we write $d x$ instead of $d(x)$ if no confusion can arise.

Lemma 4. Let $\mathbf{R}=\left(R,+, \cdot{ }^{\prime}, 0,1\right)$ be a tukasiewicz semiring, $a, b \in R$ and $d, d_{1}, d_{2} \in \operatorname{Der} \mathbf{R}$. Then

(i) $\bar{d}_{a} \in \operatorname{Der} \mathbf{R}$,

(ii) $d 0=0$,

(iii) $d a \leq a$,

(iv) $a \leq b$ implies $d a \leq d b$,

(v) $a \cdot(d 1) \leq d a$,

(vi) $d 1=1$ if and only if $d=\mathrm{id}$,

(vii) $\left(d_{1} a\right) \cdot\left(d_{2} b\right)+\left(d_{1} b\right) \cdot\left(d_{2} a\right) \leq\left(d_{1} \circ d_{2}\right)(a \cdot b)$ where $\circ$ denotes the composition of mappings,

(viii) $d_{1} \circ d_{2} \in \operatorname{Der} \mathbf{R}$ if and only if for all $x, y \in R$ we have $\left(d_{1} x\right) \cdot\left(d_{2} y\right)+\left(d_{1} y\right) \cdot\left(d_{2} x\right) \leq\left(\left(d_{1} \circ d_{2}\right) x\right) \cdot y+x \cdot\left(\left(d_{1} \circ d_{2}\right) y\right)$.

Proof.

(i) This is clear.

(ii) We have $d 0=d(0 \cdot 0)=(d 0) \cdot 0+0 \cdot(d 0)=0$.

(iii) According to (ii) and Lemma 1 (ii) we have

$$
(d a) \cdot a^{\prime} \leq d\left(a \cdot a^{\prime}\right)=d 0=0
$$

and hence $(d a) \cdot a^{\prime}=0$ whence $d a \leq a$ according to Lemma 1 (vi).

(iv) $a \leq b$ implies $d a \leq d a+d b=d(a+b)=d b$.

(v) We have $a \cdot(d 1) \leq d(a \cdot 1)=d a$.

(vi) If $d 1=1$ then $a=a \cdot(d 1) \leq d(a \cdot 1)=d a \leq a$ according to (iii) showing $d=\mathrm{id}$. The converse is trivial.

(vii) We have

$$
\begin{aligned}
& \left(d_{1} a\right) \cdot\left(d_{2} b\right)+\left(d_{1} b\right) \cdot\left(d_{2} a\right) \leq \\
& \leq\left(d_{1} a\right) \cdot\left(d_{2} b\right)+\left(d_{1} b\right) \cdot\left(d_{2} a\right)+\left(\left(d_{1} \circ d_{2}\right) a\right) \cdot b+a \cdot\left(\left(d_{1} \circ d_{2}\right) b\right)= \\
& =\left(d_{1}\left(d_{2} a\right)\right) \cdot b+\left(d_{2} a\right) \cdot\left(d_{1} b\right)+\left(d_{1} a\right) \cdot\left(d_{2} b\right)+a \cdot\left(d_{1}\left(d_{2} b\right)\right)= \\
& =d_{1}\left(\left(d_{2} a\right) \cdot b\right)+d_{1}\left(a \cdot\left(d_{2} b\right)\right)=d_{1}\left(\left(d_{2} a\right) \cdot b+a \cdot\left(d_{2} b\right)\right)=d_{1}\left(d_{2}(a \cdot b)\right)= \\
& =\left(d_{1} \circ d_{2}\right)(a \cdot b) .
\end{aligned}
$$

(viii) According to the proof of (vii) we have

$\left(d_{1} \circ d_{2}\right)(a \cdot b)=\left(d_{1} a\right) \cdot\left(d_{2} b\right)+\left(d_{1} b\right) \cdot\left(d_{2} a\right)+\left(\left(d_{1} \circ d_{2}\right) a\right) \cdot b+a \cdot\left(\left(d_{1} \circ d_{2}\right) b\right)$.

Lemma 5. Let $\mathbf{R}=\left(R,+, \cdot{ }^{\prime}, 0,1\right)$ be a tukasiewicz semiring and $d \in \operatorname{Der} \mathbf{R}$. Then the following are equivalent:

(i) $d$ is principal,

(ii) $d x=x \cdot(d 1)$ for all $x \in R$, i.e. $d=\bar{d}_{d 1}$. 
Moreover, if $x \wedge(d 1) \leq x \cdot(d 1)$ for all $x \in R$ then $d$ is principal.

Proof. The equivalence of (i) and (ii) is clear. If, finally, $x \wedge(d 1) \leq x \cdot(d 1)$ for all $x \in R$ then

$$
d x \leq x \wedge(d 1) \leq x \cdot(d 1) \leq d(x \cdot 1)=d x
$$

for all $x \in R$ and hence $d=\bar{d}_{d 1}$.

Lemma 5 shows that the Łukasiewicz semiring from Example 3 has only principal derivations since in this semiring multiplication coincides with lattice meet.

It was shown in [10], Lemma 5, that basic algebras have only principal derivations. We are going to show that this is not the case for Łukasiewicz semirings. In these semirings there exist non-principal derivations, see the following example.

Example 4. Consider the Eukasiewicz semiring $\mathbf{R}=\left(\{0, a, b, c, 1\},+, \cdot{ }^{\prime}, 0,1\right)$ with the following operations:

\begin{tabular}{|c|c|c|c|c|c|c|c|c|c|c|c|c|c|c|c|c|}
\hline+ & 0 & $a$ & $\begin{array}{ll}b & c\end{array}$ & $\begin{array}{ll}c & 1\end{array}$ & $\cdot$ & 0 & $a$ & $b$ & $c$ & 1 & & & & & & \\
\hline 0 & 0 & $a$ & $b \quad c$ & $\begin{array}{|ll|}c & 1\end{array}$ & 0 & 0 & 0 & 0 & 0 & 0 & & & & & & \\
\hline$a$ & $a$ & $a$ & $b \quad c$ & $\begin{array}{ll}c & 1\end{array}$ & $a$ & 0 & 0 & 0 & 0 & $a$ & $x$ & 0 & $a$ & $b$ & $c$ & 1 \\
\hline$b$ & $b$ & $b$ & $\begin{array}{ll}b & c\end{array}$ & $\begin{array}{ll}c & 1\end{array}$ & $b$ & 0 & 0 & 0 & $a$ & $b$ & $x^{\prime}$ & 1 & $c$ & $b$ & $a$ & 1 \\
\hline$c$ & $c$ & $c$ & $c \quad c$ & $\begin{array}{ll}c & 1\end{array}$ & $c$ & 0 & 0 & $a$ & $b$ & $c$ & & & & & & \\
\hline 1 & 1 & 1 & 11 & $\begin{array}{ll}1 & 1\end{array}$ & 1 & 0 & $a$ & $b$ & $c$ & 1 & & & & & & \\
\hline
\end{tabular}

The principal derivations look as follows:

\begin{tabular}{c|ccccc}
$x$ & 0 & $a$ & $b$ & $c$ & 1 \\
\hline $\bar{d}_{0} x$ & 0 & 0 & 0 & 0 & 0 \\
$\bar{d}_{a} x$ & 0 & 0 & 0 & 0 & $a$ \\
$\bar{d}_{b} x$ & 0 & 0 & 0 & $a$ & $b$ \\
$\bar{d}_{c} x$ & 0 & 0 & $a$ & $b$ & $c$ \\
$\bar{d}_{1} x$ & 0 & $a$ & $b$ & $c$ & 1
\end{tabular}

However, there exists a derivation $d$ of $\mathbf{R}$ which is not principal, namely the following:

$$
\begin{array}{c|ccccc}
x & 0 & a & b & c & 1 \\
\hline d x & 0 & 0 & 0 & a & a
\end{array}
$$

The Łukasiewicz semiring considered in Example 4 is isomorphic to the Łukasiewicz semiring $\mathbf{R}_{4}$ from Example 2. The following proposition shows that the situation described in Example 4 can be generalized to $\mathbf{R}_{n}$ for arbitrary $n>1$.

Proposition 1. For each $n>1$ the Eukasiewicz semiring $\mathbf{R}_{n}$ has the non-principal derivation d defined by

$$
d(x):= \begin{cases}0 & \text { if } x<n-1 \\ 1 & \text { otherwise }\end{cases}
$$


Proof. Let $a, b \in\{0, \ldots, n\}$. Then $d(a \vee b)=(d a) \vee(d b)$ since $(\{0,1, \ldots, n\}, \leq)$ is a chain. Moreover,

$$
\begin{aligned}
& d(a \odot b)= \begin{cases}0 & \text { if } a+b<2 n-1 \\
1 & \text { otherwise }\end{cases} \\
& (d a) \odot b= \begin{cases}0 & \text { if } a<n-1 \text { or } b<n \\
1 & \text { if } a \geq n-1 \text { and } b=n\end{cases} \\
& a \odot(d b)= \begin{cases}0 & \text { if } a<n \text { or } b<n-1 \\
1 & \text { if } a=n \text { and } b \geq n-1 .\end{cases}
\end{aligned}
$$

Hence $d \in \operatorname{Der} \mathbf{R}_{n}$. Since

$$
\bar{d}_{a}(b)= \begin{cases}0 & \text { if } b \leq n-a \\ b-(n-a) & \text { otherwise }\end{cases}
$$

$d$ is not principal.

For the unary operation', we can prove the following theorem:

Theorem 3. Let $\mathbf{R}=\left(R,+, \cdot{ }^{\prime}, 0,1\right)$ be a tukasiewicz semiring and $d \in \operatorname{Der} \mathbf{R}$. Then

(i) $d x^{\prime}=(d x)^{\prime}$ for all $x \in R$ if and only if $d=\mathrm{id}$,

(ii) $(d x) \cdot\left(d x^{\prime}\right) \approx x \cdot\left(d x^{\prime}\right) \approx x^{\prime} \cdot(d x) \approx 0$ and hence $d x^{\prime} \leq(d x)^{\prime}$ for all $x \in R$,

(iii) if $a \in \operatorname{Bool} \mathbf{R}$ then $d a+d a^{\prime}=d 1$,

(iv) $d\left((d 1)^{\prime}\right) \leq(d 1) \wedge(d 1)^{\prime}$,

(v) if $d 1 \in \operatorname{Bool} \mathbf{R}$ then $d\left((d 1)^{\prime} \cdot x\right)=0$ for each $x \in R$.

Proof.

(i) If $d x^{\prime}=(d x)^{\prime}$ for all $x \in R$ then for all $x \in R$ we have $x=\left(x^{\prime}\right)^{\prime} \leq\left(d x^{\prime}\right)^{\prime}=$ $\left((d x)^{\prime}\right)^{\prime}=d x \leq x$ according to Lemma 4 (iii) and hence $d=$ id.

(ii) Due to Lemma 4 (iii) and Lemma 1 (iii) and (ii) we have $(d x)\left(d x^{\prime}\right) \leq x$. $\left(d x^{\prime}\right) \leq x \cdot x^{\prime}=0$ and $x^{\prime} \cdot(d x) \leq x^{\prime} \cdot x=0$ for all $x \in R$. By Lemma 1 (vi), $d x^{\prime} \leq(d x)^{\prime}$ for all $x \in R$.

(iii) If $a \in \operatorname{Bool} \mathbf{R}$ then $d a+d a^{\prime}=d\left(a+a^{\prime}\right)=d 1$.

(iv) Since $(d 1)^{\prime} \leq 1$, we have $d\left((d 1)^{\prime}\right) \leq d 1$ by Lemma 4 (iv). According to Lemma 4 (iii) we have $d\left((d 1)^{\prime}\right) \leq(d 1)^{\prime}$. This shows $d\left((d 1)^{\prime}\right) \leq(d 1) \wedge(d 1)^{\prime}$.

(v) If $d 1 \in$ Bool R then by (iv), Lemma 4 (iv) and Lemma 1 (ii) we have $d\left((d 1)^{\prime}\right.$. $x)=\left(d\left((d 1)^{\prime}\right)\right) \cdot x+(d 1)^{\prime} \cdot(d x)=0 \cdot x+(d 1)^{\prime} \cdot(d x)=(d x) \cdot(d 1)^{\prime} \leq$ $(d 1)(d 1)^{\prime}=0$ for all $x \in R$.

Theorem 4. Let $\mathbf{R}=\left(R,+, \cdot{ }^{\prime}, 0,1\right)$ be a Eukasiewicz semiring, $a \in R$ and $d \in$ Der R. Then

(i) $(\operatorname{Der} \mathbf{R},+)$ is a join-semilattice with the least element $\bar{d}_{0}$ and the greatest element id, 
(ii) Fix $d$ is a subuniverse of $(R,+, \cdot, 0)$,

(iii) $($ Fix $d) \cap d^{-1}(\{0\})=\{0\}$,

(iv) if $a \in \operatorname{Bool} \mathbf{R}$ then $\bar{d}_{a} \in \operatorname{End}(R,+, \cdot, 0)$.

Proof.

(i) It is easy to see that $\operatorname{Der} \mathbf{R}$ is closed with respect to + . Hence $(\operatorname{Der} \mathbf{R},+)$ is a semilattice which is considered as a join-semilattice. We then have $d_{1} \leq d_{2}$ if and only if $d_{1} x \leq d_{2} x$ for all $x \in R$. Obviously, $\bar{d}_{0}$, id $\in \operatorname{Der} \mathbf{R}$ and $\bar{d}_{0}$ is the smallest element of (Der $\mathbf{R}, \leq$ ). Because of Lemma 4 (iii), id is the greatest element of (Der $\mathbf{R}, \leq)$.

(ii) If $a, b \in$ Fix $d$ then $d(a+b)=d a+d b=a+b$ and

$$
d(a \cdot b)=(d a) \cdot b+a \cdot(d b)=a \cdot b+a \cdot b=a \cdot b .
$$

Moreover, $d 0=0$ according to Lemma 4 (ii).

(iii) $a \in($ Fix $d) \cap d^{-1}(\{0\})$ implies $a=d a=0$.

(iv) This can be proved by a straightforward computation.

For Łukasiewicz semirings we adopt the concept of an ideal as introduced for semirings in [9]. Hence, we define

Definition 3. Let $\mathbf{R}=\left(R,+, \cdot,{ }^{\prime}, 0,1\right)$ be a tukasiewicz semiring. An ideal of $\mathbf{R}$ is a subset $I$ of $R$ satisfying

$$
\begin{aligned}
& 0 \in I, \\
& \text { if } x, y \in I \text { then } x+y \in I, \\
& \text { if } x \in I \text { and } y \in R \text { then } x \cdot y \in I .
\end{aligned}
$$

Let Id $\mathbf{R}$ denote the set of all ideals of $\mathbf{R}$.

It is well known and easy to prove that $(\operatorname{Id} \mathbf{R}, \subseteq)$ is a complete lattice the with the smallest element $\{0\}$ and the greatest element $R$.

Proposition 2. Let $\mathbf{R}=\left(R,+, \cdot{ }^{\prime}, 0,1\right)$ be a Eukasiewicz semiring, $d \in \operatorname{Der} \mathbf{R}$ and $I \in \operatorname{Id} \mathbf{R}$. Then

(i) $d^{-1}(\{0\}) \in \operatorname{Id} \mathbf{R}$,

(ii) $d^{-1}(I):=\{x \in R \mid d x \in I\}$ is a subuniverse of $(R,+, \cdot, 0)$.

Proof.

(i) We have $0 \in d^{-1}(\{0\})$. Moreover, $d^{-1}(\{0\})$ is closed with respect to + . If $a \in d^{-1}(\{0\})$ and $b \in R$ then $d(a \cdot b) \leq d(a \cdot 1)=d a=0$ according to Lemma 1 (iii) and Lemma 4 (iv) and hence $d(a \cdot b)=0$, i.e. $a \cdot b \in d^{-1}(\{0\})$.

(ii) The proof is straightforward. 
Let $\mathbf{R}=\left(R,+, \cdot{ }^{\prime}, 0,1\right)$ be a Łukasiewicz semiring and $\Theta \in$ Con $\mathbf{R}$. Denote by $[0] \Theta$ the class of 0 with respect to $\Theta$, the so-called kernel of $\Theta$. It is well-known that in any semiring, the kernel of any congruence is an ideal, but not every ideal is a kernel of some congruence, see e.g. [9]. Now let $d \in \operatorname{Der} \mathbf{R}$. We are interested in the question if $d^{-1}([0] \Theta) \in \operatorname{Id} \mathbf{R}$.

Theorem 5. Let $\mathbf{R}=\left(R,+, \cdot{ }^{\prime}, 0,1\right)$ be a Łukasiewicz semiring, $\Theta \in \operatorname{Con} \mathbf{R}$ and $d \in \operatorname{Der} \mathbf{R}$. Then

(i) $d^{-1}([0] \Theta):=\{x \in R \mid d x \in[0] \Theta\} \in \operatorname{Id} \mathbf{R}$,

(ii) $d([0] \Theta) \subseteq[0] \Theta$ and $d([0] \Theta)$ is a subuniverse of $(R,+, 0)$.

Proof.

(i) If $I:=d^{-1}([0] \Theta), a, b \in I$ and $c \in R$ then $d a, d b \in[0] \Theta$ and hence

$$
\begin{aligned}
d(a+b) & =d a+d b \in[0] \Theta, \\
d(a \cdot c) & =d(a \cdot c)+0 \in[d(a \cdot c)+d a] \Theta=[d a] \Theta=[0] \Theta
\end{aligned}
$$

according to Lemma 1 (iii) and Lemma 4 (iv) showing $a+b, a \cdot c \in I$.

(ii) Clearly, $0=d 0 \in d([0] \Theta)$. If $a, b \in d([0] \Theta)$ then there exist $c, e \in[0] \Theta$ with $d c=a$ and $d e=b$ and hence

$$
\begin{aligned}
a & =d c=d c+0 \in[d c+c] \Theta=[c] \Theta=[0] \Theta, \\
a+b & =d c+d e=d(c+e) \in d([0] \Theta)
\end{aligned}
$$

according to Lemma 4 (iii).

Theorem 6. Let $\mathbf{R}=\left(R,+, \cdot{ }^{\prime}, 0,1\right)$ be a tukasiewicz semiring, $d \in \operatorname{Der} \mathbf{R}$, assume $d 1 \in$ Bool $\mathbf{R}$, put

$$
\begin{aligned}
x^{*}: & =x^{\prime} \wedge(d 1), \\
x \cap y & :=\left(x^{*}+y^{*}\right)^{*}
\end{aligned}
$$

for all $x, y \in d(R)$ and assume $x^{*} \in d(R)$ for all $x \in d(R)$. Then $\left(d(R),+, \cap,{ }^{*}, 0, d 1\right)$ is a bounded lattice with an antitone involution.

Proof. Since $d 1 \in$ Bool R, i.e. $(d 1) \wedge(d 1)^{\prime}=0$, we have

$$
\begin{aligned}
\left(x^{*}\right)^{*} & =\left(x^{\prime} \wedge(d 1)\right)^{\prime} \wedge(d 1)=\left(x+(d 1)^{\prime}\right) \wedge(d 1) \\
& =(x \wedge(d 1))+\left((d 1)^{\prime} \wedge(d 1)\right)=x+0=x
\end{aligned}
$$

for all $x \in d(R)$. Obviously, ${ }^{*}$ is antitone. The rest of the proof is clear.

Theorem 7. Let $\mathbf{R}=\left(R,+, \cdot{ }^{\prime}, 0,1\right)$ and $\mathbf{S}=\left(S,+, \cdot,{ }^{\prime}, 0,1\right)$ be Eukasiewicz semirings. Then $\operatorname{Der}(\mathbf{R} \times \mathbf{S})=\operatorname{Der} \mathbf{R} \times \operatorname{Der} \mathbf{S}$. 
Proof. Clearly, $\operatorname{Der} \mathbf{R} \times \operatorname{Der} \mathbf{S} \subseteq \operatorname{Der}(\mathbf{R} \times \mathbf{S})$. In order to prove the converse inclusion, let $d \in \operatorname{Der}(\mathbf{R} \times \mathbf{S})$. Then for each $x \in R$ we have $d(x, 0) \leq(x, 0)$ according to Lemma 4 (iii) and hence there exists some mapping $d_{1}$ from $R$ to $R$ satisfying $\left(d_{1} x, 0\right)=d(x, 0)$ for all $x \in R$. Analogously, there exists some mapping $d_{2}$ from $S$ to $S$ satisfying $\left(0, d_{2} y\right)=d(0, y)$ for all $y \in S$. Now let $a, b \in R$ and $c \in S$. Then

$$
\begin{aligned}
\left(d_{1}(a+b), 0\right) & =d(a+b, 0)=d((a, 0)+(b, 0))=d(a, 0)+d(b, 0)= \\
& =\left(d_{1} a, 0\right)+\left(d_{1} b, 0\right)=\left(d_{1} a+d_{1} b, 0\right),
\end{aligned}
$$

i.e. $d_{1}(a+b)=d_{1} a+d_{1} b$. Moreover,

$$
\begin{aligned}
\left(d_{1}(a \cdot b), 0\right) & =d(a \cdot b, 0)=d((a, 0) \cdot(b, 0))=(d(a, 0)) \cdot(b, 0)+(a, 0) \cdot(d(b, 0))= \\
& =\left(d_{1} a, 0\right) \cdot(b, 0)+(a, 0) \cdot\left(d_{1} b, 0\right)=\left(\left(d_{1} a\right) \cdot b, 0\right)+\left(a \cdot\left(d_{1} b\right), 0\right)= \\
& =\left(\left(d_{1} a\right) \cdot b+a \cdot\left(d_{1} b\right), 0\right),
\end{aligned}
$$

i.e. $d_{1}(a \cdot b)=\left(d_{1} a\right) \cdot b+a \cdot\left(d_{1} b\right)$. This shows $d_{1} \in \operatorname{Der} \mathbf{R}$. Analogously, $d_{2} \in \operatorname{Der} \mathbf{S}$. Finally,

$$
d(a, c)=d((a, 0)+(0, c))=d(a, 0)+d(0, c)=\left(d_{1} a, 0\right)+\left(0, d_{2} c\right)=\left(d_{1} a, d_{2} c\right)
$$

proving $d \in \operatorname{Der} \mathbf{R} \times \operatorname{Der} \mathbf{S}$. Hence $\operatorname{Der}(\mathbf{R} \times \mathbf{S}) \subseteq \operatorname{Der} \mathbf{R} \times \operatorname{Der} \mathbf{S}$ completing the proof of the theorem.

Proposition 3. Let $\mathbf{R}=\left(R,+, \cdot,{ }^{\prime}, 0,1\right)$ be a Eukasiewicz semiring and $a \in \mathrm{Bool} \mathbf{R}$. Then $\left(\bar{d}_{a}(R),+, \cdot, 0, a\right)$ is a commutative semiring satisfying $x+x \approx x$ and $x+a \approx$ a.

Proof. Since $\bar{d}_{a}(x+y)=\bar{d}_{a} x+\bar{d}_{a} y, \bar{d}_{a}(x \cdot y)=a \cdot(x \cdot y)=(a \cdot x) \cdot(a \cdot y)=$ $\left(\bar{d}_{a} x\right) \cdot\left(\bar{d}_{a} y\right)$ for all $x, y \in R$ and $\bar{d}_{a} 0=a \cdot 0=0$ and $\bar{d}_{a} 1=a \cdot 1=a, \bar{d}_{a}$ is a homomorphism from $(R,+, \cdot, 0,1)$ onto $\left(\bar{d}_{a}(R),+, \cdot, 0, a\right)$.

\section{4. $(f, g)$-DERIVATIONS}

The concept of an $(f, g)$-derivation is mentioned in the monograph [9]. For Łukasiewicz semirings, it is specified as follows:

Definition 4. Let $\mathbf{R}=\left(R,+, \cdot{ }^{\prime}, 0,1\right)$ be a Łukasiewicz semiring and $f, g \in$ End $\mathbf{R}$. An $(f, g)$-derivation on $\mathbf{R}$ is a mapping $d$ from $R$ to $R$ satisfying the following identities:

$$
\begin{aligned}
d(x+y) & \approx d x+d y, \\
d(x \cdot y) & \approx(d x) \cdot f(y)+g(x) \cdot(d y) .
\end{aligned}
$$

Let $\operatorname{Der}_{f, g} \mathbf{R}$ denote the set of all $(f, g)$-derivations on $\mathbf{R}$. Especially, $\operatorname{Der}_{\text {id, id }} \mathbf{R}=$ DerR.

The following example shows that there exist $(f, g)$-derivations on Łukasiewicz semirings which are not derivations. 
Example 5. Let $M$ be a set with $|M|>1$ and put $\mathbf{R}:=\left(2^{M}, \cup, \cap,{ }^{\prime}, \varnothing, M\right)$. If $f \in$ End $\mathbf{R}$ then $f \in \operatorname{Der}_{f, f} \mathbf{R}$ since

$$
\begin{aligned}
& f(A \cup B)=f(A) \cup f(B), \\
& f(A \cap B)=f(A \cap B) \cup f(A \cap B)=(f(A) \cap f(B)) \cup(f(A) \cap f(B))
\end{aligned}
$$

for all $A, B \in 2^{M}$. Now let $a \in M$ and $g$ denote the mapping from $2^{M}$ to $2^{M}$ defined by

$$
g(A):=\left\{\begin{array}{cl}
M & \text { if } a \in A, \\
\varnothing & \text { otherwise }
\end{array}\right.
$$

$\left(A \in 2^{M}\right.$ ). Then $g \in \operatorname{End} \mathbf{R}$ (and hence $g \in \operatorname{Der}_{g, g} \mathbf{R}$ ) as can be seen from the following table:

\begin{tabular}{c|c|c|c|c|c|c}
$A$ & $B$ & $g(A)$ & $g(B)$ & $g(A \cup B)$ & $g(A \cap B)$ & $g\left(A^{\prime}\right)$ \\
\hline$\ni a$ & $\ni a$ & $M$ & $M$ & $M$ & $M$ & $\varnothing$ \\
\hline$\ni a$ & $\ngtr a a$ & $M$ & $\varnothing$ & $M$ & $\varnothing$ & $\varnothing$ \\
\hline$\not \supset a$ & $\ni a$ & $\varnothing$ & $M$ & $M$ & $\varnothing$ & $M$ \\
\hline$\not \supset a$ & $\ngtr a a$ & $\varnothing$ & $\varnothing$ & $\varnothing$ & $\varnothing$ & $M$
\end{tabular}

But $g \notin$ Der $\mathbf{R}$ since

$$
g(\{a\} \cap\{x\})=\varnothing \neq\{x\}=(g(\{a\}) \cap\{x\}) \cup(\{a\} \cap g(\{x\}))
$$

for all $x \in M \backslash\{a\}$.

Lemma 6. Let $\mathbf{R}=\left(R,+, \cdot{ }^{\prime}, 0,1\right)$ be a Eukasiewicz semiring, $f, g \in$ End $\mathbf{R}, a, b \in$ $R$ and $d, d_{1}, d_{2} \in \operatorname{Der}_{f, g} \mathbf{R}$. Then

(i) $\bar{d}_{0} \in \operatorname{Der}_{f, g} \mathbf{R}$,

(ii) $d 0=0$,

(iii) $d a \leq f(a) \wedge g(a)$,

(iv) $a \leq b$ implies $d a \leq d b$,

(v) $(f(a)+g(a)) \cdot(d 1) \leq d a$,

(vi) $d 1=1$ if and only if $d=f=g$,

(vii) $\left(d_{1}(g(a))\right) \cdot f\left(d_{2} b\right)+\left(d_{1}(f(b))\right) \cdot g\left(d_{2} a\right) \leq\left(d_{1} \circ d_{2}\right)(a \cdot b)$,

(viii) if $f \circ f=f$ and $g \circ g=g$ then $d_{1} \circ d_{2} \in \operatorname{Der}_{f, g} \mathbf{R}$ if and only if for all $x, y \in R$ we have $\left(d_{1}(g(x))\right) \cdot f\left(d_{2} y\right)+\left(d_{1}(f(y))\right) \cdot g\left(d_{2} x\right) \leq\left(\left(d_{1} \circ d_{2}\right) x\right)$. $f(y)+g(x) \cdot\left(\left(d_{1} \circ d_{2}\right) y\right)$.

Proof.

(i) This is clear.

(ii) We have $d 0=d(0 \cdot 0)=(d 0) \cdot f(0)+g(0) \cdot(d 0)=(d 0) \cdot 0+0 \cdot(d 0)=0$.

(iii) According to (ii) and Lemma 1 (ii) we have

$$
(d a) \cdot(f(a))^{\prime}=(d a) \cdot f\left(a^{\prime}\right) \leq d\left(a \cdot a^{\prime}\right)=d 0=0
$$


and hence $(d a) \cdot(f(a))^{\prime}=0$ whence $d a \leq f(a)$ according to Lemma 1 (vi). Analogously, according to (ii) and Lemma 1 (ii) we have

$$
(d a) \cdot(g(a))^{\prime}=g\left(a^{\prime}\right) \cdot(d a) \leq d\left(a^{\prime} \cdot a\right)=d 0=0
$$

and hence $(d a) \cdot(g(a))^{\prime}=0$ whence $d a \leq g(a)$ according to Lemma 1 (vi).

(iv) can be proved exactly as Lemma 4 (iv).

(v) We have

$$
(f(a)+g(a)) \cdot(d 1)=(d 1) \cdot f(a)+g(a) \cdot(d 1) \leq d(1 \cdot a)+d(a \cdot 1)=d a+d a=d a .
$$

(vi) If $d 1=1$ then

$$
\begin{aligned}
& f(a)=(d 1) \cdot f(a) \leq d(1 \cdot a)=d a \leq f(a), \\
& g(a)=g(a) \cdot(d 1) \leq d(a \cdot 1)=d a \leq g(a)
\end{aligned}
$$

according to (iii) showing $d=f=g$. The converse is trivial.

(vii) We have

$$
\begin{aligned}
& \left(d_{1}(g(a))\right) \cdot f\left(d_{2} b\right)+\left(d_{1}(f(b))\right) \cdot g\left(d_{2} a\right) \leq \\
& \leq\left(d_{1}(g(a))\right) \cdot f\left(d_{2} b\right)+\left(d_{1}(f(b))\right) \cdot g\left(d_{2} a\right)+ \\
& +\left(\left(d_{1} \circ d_{2}\right) a\right) \cdot f(f(b))+g(g(a)) \cdot\left(\left(d_{1} \circ d_{2}\right) b\right)= \\
& =\left(d_{1}\left(d_{2} a\right)\right) \cdot f(f(b))+g\left(d_{2} a\right) \cdot\left(d_{1}(f(b))\right)+ \\
& +\left(d_{1}(g(a))\right) \cdot f\left(d_{2} b\right)+g(g(a)) \cdot\left(d_{1}\left(d_{2} b\right)\right)= \\
& =d_{1}\left(\left(d_{2} a\right) \cdot f(b)\right)+d_{1}\left(g(a) \cdot\left(d_{2} b\right)\right)=d_{1}\left(\left(d_{2} a\right) \cdot f(b)+g(a) \cdot\left(d_{2} b\right)\right)= \\
& =d_{1}\left(d_{2}(a \cdot b)\right)=\left(d_{1} \circ d_{2}\right)(a \cdot b) .
\end{aligned}
$$

(viii) If $f \circ f=f$ and $g \circ g=g$ then according to the proof of (vii) we have

$$
\begin{aligned}
\left(d_{1} \circ d_{2}\right)(a \cdot b) & =\left(d_{1}(g(a))\right) \cdot f\left(d_{2} b\right)+\left(d_{1}(f(b))\right) \cdot g\left(d_{2} a\right)+ \\
& +\left(\left(d_{1} \circ d_{2}\right) a\right) \cdot f(b)+g(a) \cdot\left(\left(d_{1} \circ d_{2}\right) b\right) .
\end{aligned}
$$

For the unary operation ', we can prove the following theorem:

Theorem 8. Let $\mathbf{R}=\left(R,+, \cdot{ }^{\prime}, 0,1\right)$ be a Eukasiewicz semiring, $f, g \in$ End $\mathbf{R}$ and $d \in \operatorname{Der}_{f, g} \mathbf{R}$. Then

(i) $d x^{\prime}=(d x)^{\prime}$ for all $x \in R$ if and only if $d=f=g$,

(ii) $(d x) \cdot\left(d x^{\prime}\right) \approx f(x) \cdot\left(d x^{\prime}\right) \approx f\left(x^{\prime}\right) \cdot(d x) \approx g(x) \cdot\left(d x^{\prime}\right) \approx g\left(x^{\prime}\right) \cdot(d x) \approx 0$ and hence $d x^{\prime} \leq(d x)^{\prime}$ for all $x \in R$,

(iii) if $a \in$ Bool $\mathbf{R}$ then $d a+d a^{\prime}=d 1$.

Proof. 
(i) If $d x^{\prime}=(d x)^{\prime}$ for all $x \in R$ then for all $x \in R$ we have

$$
\begin{aligned}
& f(x)=\left(f\left(x^{\prime}\right)\right)^{\prime} \leq\left(d x^{\prime}\right)^{\prime}=d x \leq f(x), \\
& g(x)=\left(g\left(x^{\prime}\right)\right)^{\prime} \leq\left(d x^{\prime}\right)^{\prime}=d x \leq g(x)
\end{aligned}
$$

according to Lemma 6 (iii) and hence $d=f=g$.

(ii) Due to Lemma 6 (iii) and Lemma 1 (iii) and (ii) we have

$$
\begin{aligned}
& (d x) \cdot\left(d x^{\prime}\right) \leq f(x) \cdot\left(d x^{\prime}\right) \leq f(x) \cdot f\left(x^{\prime}\right)=f\left(x \cdot x^{\prime}\right)=f(0)=0, \\
& f\left(x^{\prime}\right) \cdot(d x) \leq f\left(x^{\prime}\right) \cdot f(x)=f\left(x \cdot x^{\prime}\right)=f(0)=0
\end{aligned}
$$

for all $x \in R$. The proof for $g$ is analogous. By Lemma 1 (vi), $d x^{\prime} \leq(d x)^{\prime}$ for all $x \in R$.

(iii) can be proved exactly as Theorem 3 (iii).

Theorem 9. Let $\mathbf{R}=\left(R,+, \cdot{ }^{\prime}, 0,1\right)$ be a Łukasiewicz semiring, $f, g \in$ End $\mathbf{R}$ and $d \in \operatorname{Der}_{f, g} \mathbf{R}$. Then

(i) $\left(\operatorname{Der}_{f, g} \mathbf{R},+\right)$ is a join-semilattice with the least element $\bar{d}_{0}$,

(ii) $($ Fix $d) \cap d^{-1}(\{0\})=\{0\}$.

\section{Proof.}

(i) It is easy to see that $\operatorname{Der}_{f, g} \mathbf{R}$ is closed with respect to + . Hence $\left(\operatorname{Der}_{f, g} \mathbf{R},+\right)$ is a semilattice which is considered as a join-semilattice. We then have $d_{1} \leq$ $d_{2}$ if and only if $d_{1} x \leq d_{2} x$ for all $x \in R$. Obviously, $\bar{d}_{0} \in \operatorname{Der}_{f, g} \mathbf{R}$ and $\bar{d}_{0}$ is the smallest element of $\left(\operatorname{Der}_{f, g} \mathbf{R}, \leq\right)$.

(ii) can be proved exactly as Theorem 4 (iii).

It is clear that Proposition 2, Theorem 5 (i) and Theorem 6 remain valid for $(f, g)$ derivations, too.

Theorem 10. Let $\mathbf{R}=\left(R,+, \cdot,{ }^{\prime}, 0,1\right)$ and $\mathbf{S}=\left(S,+, \cdot{ }^{\prime}, 0,1\right)$ be Eukasiewicz semirings, $f_{1}, g_{1} \in \operatorname{End} \mathbf{R}$ and $f_{2}, g_{2} \in \operatorname{End} \mathbf{S}$. Then $\left(f_{1}, f_{2}\right),\left(g_{1}, g_{2}\right) \in \operatorname{End}(\mathbf{R} \times \mathbf{S})$ and

$$
\operatorname{Der}_{\left(f_{1}, f_{2}\right),\left(g_{1}, g_{2}\right)}(\mathbf{R} \times \mathbf{S})=\operatorname{Der}_{f_{1}, g_{1}} \mathbf{R} \times \operatorname{Der}_{f_{2}, g_{2}} \mathbf{S} .
$$

Proof. Clearly, $\left(f_{1}, f_{2}\right),\left(g_{1}, g_{2}\right) \in \operatorname{End}(\mathbf{R} \times \mathbf{S})$ and

$$
\operatorname{Der}_{f_{1}, g_{1}} \mathbf{R} \times \operatorname{Der}_{f_{2}, g_{2}} \mathbf{S} \subseteq \operatorname{Der}_{\left(f_{1}, f_{2}\right),\left(g_{1}, g_{2}\right)}(\mathbf{R} \times \mathbf{S}) .
$$

In order to prove the converse inclusion, let $d \in \operatorname{Der}_{\left(f_{1}, f_{2}\right),\left(g_{1}, g_{2}\right)}(\mathbf{R} \times \mathbf{S})$. Then for each $x \in R$ we have $d(x, 0) \leq\left(f_{1}(x), 0\right)$ according to Lemma 6 (iii) and hence there exists some mapping $d_{1}$ from $R$ to $R$ satisfying $\left(d_{1} x, 0\right)=d(x, 0)$ for all $x \in R$. 
Analogously, there exists some mapping $d_{2}$ from $S$ to $S$ satisfying $\left(0, d_{2} y\right)=d(0, y)$ for all $y \in S$. Now let $a, b \in R$ and $c \in S$. Then

$$
\begin{aligned}
\left(d_{1}(a+b), 0\right) & =d(a+b, 0)=d((a, 0)+(b, 0))=d(a, 0)+d(b, 0)= \\
& =\left(d_{1} a, 0\right)+\left(d_{1} b, 0\right)=\left(d_{1} a+d_{1} b, 0\right),
\end{aligned}
$$

i.e. $d_{1}(a+b)=d_{1} a+d_{1} b$. Moreover,

$$
\begin{aligned}
\left(d_{1}(a \cdot b), 0\right) & =d(a \cdot b, 0)=d((a, 0) \cdot(b, 0))= \\
& =(d(a, 0)) \cdot\left(f_{1}(b), 0\right)+\left(g_{1}(a), 0\right) \cdot(d(b, 0))= \\
& =\left(d_{1} a, 0\right) \cdot\left(f_{1}(b), 0\right)+\left(g_{1}(a), 0\right) \cdot\left(d_{1} b, 0\right)= \\
& =\left(\left(d_{1} a\right) \cdot f_{1}(b), 0\right)+\left(g_{1}(a) \cdot\left(d_{1} b\right), 0\right)= \\
& =\left(\left(d_{1} a\right) \cdot f_{1}(b)+g_{1}(a) \cdot\left(d_{1} b\right), 0\right),
\end{aligned}
$$

i.e. $d_{1}(a \cdot b)=\left(d_{1} a\right) \cdot f_{1}(b)+g_{1}(a) \cdot\left(d_{1} b\right)$. This shows $d_{1} \in \operatorname{Der}_{f_{1}, g_{1}} \mathbf{R}$. Analogously, $d_{2} \in \operatorname{Der}_{f_{2}, g_{2}}$ S. Finally,

$d(a, c)=d((a, 0)+(0, c))=d(a, 0)+d(0, c)=\left(d_{1} a, 0\right)+\left(0, d_{2} c\right)=\left(d_{1} a, d_{2} c\right)$

proving $d \in \operatorname{Der}_{f_{1}, g_{1}} \mathbf{R} \times \operatorname{Der}_{f_{2}, g_{2}} \mathbf{S}$. Hence

$$
\operatorname{Der}_{\left(f_{1}, f_{2}\right),\left(g_{1}, g_{2}\right)}(\mathbf{R} \times \mathbf{S}) \subseteq \operatorname{Der}_{f_{1}, g_{1}} \mathbf{R} \times \operatorname{Der}_{f_{2}, g_{2}} \mathbf{S}
$$

completing the proof of the theorem.

\section{ACKNOWLEDGEMENT}

We thank the referee for his valuable suggestions which made the paper better readable.

\section{REFERENCES}

[1] N. O. Alshehri, "Derivations of MV-algebras," Int. J. Math. Math. Sci, vol. 2010, p. 7, 2010, doi: 10.1155/2010/312027.

[2] L. P. Belluce, A. Di Nola, and A. R. Ferraioli, "MV-semirings and their sheaf representations," Order, vol. 30, no. 1, pp. 165-179, 2013.

[3] S. Bonzio, I. Chajda, and A. Ledda, "Representing quantum structures as near semirings," Log. J. IGPL, vol. 24, no. 5, pp. 719-742, 2016.

[4] I. Chajda, D. Fazio, and A. Ledda, "A semiring-like representation of lattice pseudoeffect algebras," Soft Computing, 2018, doi: https://doi.org/10.1007/s00500-018-3157-2.

[5] I. Chajda, D. Fazio, and A. Ledda, "On the structure theory of Łukasiewicz near semirings," Log. J. IGPL, vol. 26, no. 1, pp. 14-28, 2018, doi: https://doi.org/10.1093/jigpal/jzx044.

[6] I. Chajda and H. Länger, "A representation of lattice effect algebras by means of right near semirings with involution," Int. J. Theor. Phys, vol. 56, no. 12, pp. 3719-3726, 2017, doi: 10.1007/s10773-016-3191-8.

[7] L. Ferrari, "On derivations of lattices," PU.M.A., Pure Math. Appl, vol. 12, no. 4, pp. 365-382, 2001.

[8] B. Gerla, "Many-valued logic and semirings," Neural Network World, vol. 13, pp. 467-480, 2003.

[9] J. S. Golan, Semirings and their applications. Dordrecht: Kluwer Academic Publishers, 1999. 
[10] J. Krňávek and J. Kühr, “A note on derivations on basic algebras,” Soft Comput, vol. 19, no. 7, pp. 1765-1771, 2015, doi: 10.1007/s00500-014-1586-0.

[11] G. Szasz, "Derivations of lattices," Acta Sci. Math, vol. 37, pp. 149-154, 1975.

\section{Authors' addresses}

\section{Ivan Chajda}

Palacký University Olomouc, Faculty of Science, Department of Algebra and Geometry, 17. listopadu 12, 77146 Olomouc, Czech Republic

E-mail address: ivan.chajda@upol.cz

\section{Helmut Länger}

TU Wien, Faculty of Mathematics and Geoinformation, Institute of Discrete Mathematics and Geometry, Wiedner Hauptstraße 8-10, 1040 Vienna, Austria, and, Palacký University Olomouc, Faculty of Science, Department of Algebra and Geometry, 17. listopadu 12, 77146 Olomouc, Czech Republic E-mail address: helmut. laenger@tuwien.ac.at 